\title{
Design of an Embedded Control System Laboratory Experiment
}

\author{
Pau Martí, Member, IEEE, Manel Velasco, Josep M. Fuertes, Member, IEEE, \\ Antonio Camacho, and Giorgio Buttazzo, Senior Member, IEEE
}

\begin{abstract}
This paper presents a prototype laboratory experiment to be integrated in the education of embedded control system engineers. The experiment, a real-time control of a dynamical system, is designed to drive students to a deeper understanding and integration of the diverse theoretical concepts that often come from different disciplines such as real-time systems and control systems. Rather than proposing the experiment for a particular course within an embedded system engineering curriculum, this paper describes how the experiment can be tailored to the needs and diverse background of both undergraduate and graduate students education.
\end{abstract}

Index Terms-Control systems, embedded system education, laboratory experiment, real-time systems.

\section{INTRODUCTION}

$\mathbf{T}$ HE economic importance of embedded systems has grown exponentially as electronic components are in everyday-use devices. Hence, embedded system education is a strategic asset, and university curricula are being adapted accordingly to cover this domain [1]. Embedded system courses are being integrated into existing science and engineering curricula [2], [3], but also, specific curricula have been developed to integrate the broad set of concepts into a course sequence [4]-[7]. In addition, modern teaching practices, such as problem-based learning [8], international project collaboration [9], cooperative learning [10], online competitions [11], educational games [12], or remote laboratories [13], have also been applied to the embedded system education. To provide students with in-depth understanding across all the areas and disciplines involved in embedded systems is a difficult task. Hence, laboratory activities are crucial to consolidate the diverse theoretical material [14].

Since many embedded systems are control systems [15] and considering that there is an increasing trend to adopt real-time technology for the embedded computing platform [16], laboratory experiments, including topics of real-time and

Manuscript received March 10, 2009; revised June 15, 2009; accepted December 23, 2009. Date of publication February 8, 2010; date of current version September 10, 2010. This work was supported in part by ArtistDesign NoE IST-FP7-2008-214373 and in part by Spanish Ministerio de Educación y Ciencia Project CICYT DPI2007-61527.

P. Martí, M. Velasco, J. M. Fuertes, and A. Camacho are with the Department of Automatic Control, Technical University of Catalonia (UPC), 08028 Barcelona, Spain (e-mail: pau.marti@upc.edu; manel.velasco@upc.edu; josep. m.fuertes@upc.edu; antonio.camacho.santiago@upc.edu).

G. Buttazzo is with the Real-Time Systems Laboratory, Computer Engineering Department, Scuola Superiore Sant'Anna, 56124 Pisa, Italy (e-mail: giorgio@sssup.it).

Color versions of one or more of the figures in this paper are available online at http://ieeexplore.iee.org.

Digital Object Identifier 10.1109/TIE.2010.2040559 control systems, are becoming more and more important for the education of embedded system engineers. The traditional teaching approach to real-time system and to control system courses can be quite math intensive and abstract, thus failing to introduce students to the realities of embedded control system implementation. Moreover, the natural interaction and integration between these two disciplines is often neglected due to the traditional compartmentalized nature of science and engineering education. Since control systems are real-time systems, control engineers must have an understanding of computers and real-time systems, while computer engineers must understand control theory.

To overcome such limitations, this paper presents a laboratory experiment to be integrated in the education of embedded control system engineers that flexibly combines two main disciplines: real-time systems and control systems. The flexibility is achieved by describing a set of problems/observations that provide the spectrum of possible choices that instructors/ students have and the work that has to be done to complete the experiment. This permits elaborating diverse assignments for the laboratory experiment with open problems, rather than providing tight guidelines, while providing the tools for assessing whether the students' design and implementation choices were correct. Finally, through the experiment, it is shown that the combination of both disciplines does not rise conflicts. It rather provides complementary approaches/views that help in the multidisciplinary learning process required in the embedded system education.

The experiment main activity includes the implementation of a real-time control application, consisting in controlling a physical plant by a controller implemented as a software task executing on top of a real-time operating system (RTOS). Rather than proposing an experiment for a particular course within an embedded system engineering curriculum, this paper describes how the experiment can be tailored to the needs of both undergraduate and graduate students education and to the diverse background of the target audience. A tentative laboratory program covering the different stages required to carry out the experiment is presented, and its integration into a master-level student course is also reported.

The rest of this paper is organized as follows. Section II sets the objectives, competence, and learning outcomes for the designed experiment. Section III discusses the selection of the controlled plant and the processing platform/RTOS. Section IV introduces the set of problems/observations for carrying out the activity. Section V presents an outline of the experiment and its adaptation to a course. Section VI concludes this paper. 
TABLE I

REQUIRED SKILLS

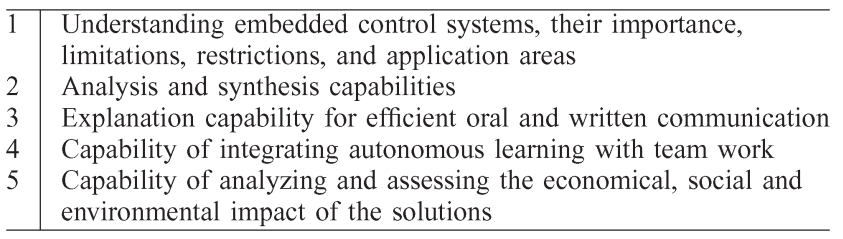

TABLE II

LEARNING OUTCOMES

\begin{tabular}{l|l}
2 & Identify embedded systems features \\
3 & Identify components, concepts and design methodologies \\
4 & Interpret data-sheets, documentation and specifications \\
5 & Pesign, build and troubleshoot an embedded control system \\
6 & Practice on modelling, analysis and design of control systems \\
7 & Evaluate system performance \\
\hline
\end{tabular}

\section{Objectives, Competence, AND LEARNING OUTCOMES}

The experiment objectives are twofold. First, the positive benefits of experimental learning are well known in educational and professional activities. Students' confidence and enthusiasm in experiments grow as they practice in problem solving, teamwork, design skills, etc. Second and more specifically, experiments should educate students in embedded control systems, providing additional knowledge that they cannot acquire from theory.

Looking at the European Credit Transfer System, program objectives are preferably specified in terms of the learning outcomes and competence to be acquired. Although the proposed experiment is neither tailored to a specific program nor to any specific level of study (undergraduate and graduate), the main skills to be acquired by students are listed in Table I. Skills 1 and 2 relate to technical aspects and theoretical knowledge on embedded control systems; skills 3 and 4 relate to practical issues and experimental learning, whereas skill 5 refers to sustainability issues. The multidisciplinary nature of embedded systems requires more background and transversal knowledge in different fields, combined with the capability of integrating different skills for a system-wide objective. The learning outcomes are more specific because they state what is expected from a student as a result of the learning process.

The learning outcomes are listed in Table II. The first three outcomes are related to understanding embedded control systems, from a technical point of view, taking into account the multidisciplinary nature of the field. In this process, it is also crucial for students to be able to read, understand, and use existing documentation, like datasheets, application programming interface (API) reference manuals, etc. Outcomes 4-6 are related to implementation issues, which are essential for reproducing an experiment. Finally, the evaluation of system performance is crucial to assess if the specifications are met.

The required background for students to carry out the experiments is a basic knowledge on control system theory and real-time programming using an operating system.

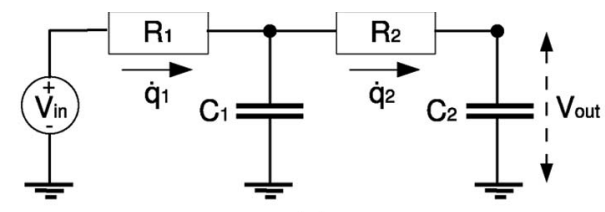

(a)

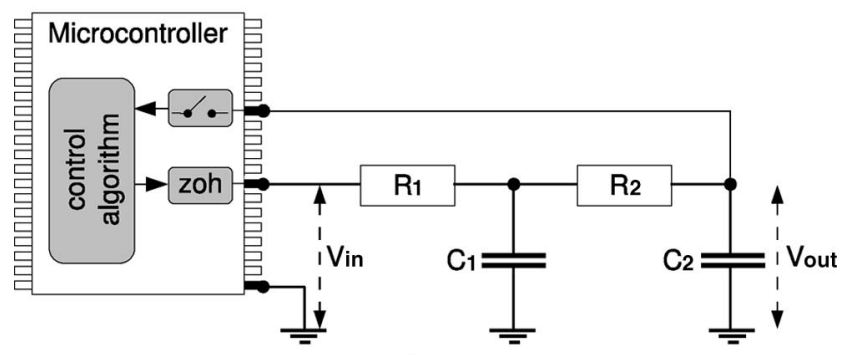

(b)

Fig. 1. Plant and control setup. (a) $R C R C$ circuit. (b) Control setup.

\section{Selection of the Controlled Plant And PROCESSING PLATFORM}

The controlled plant and processing platform (hardware and RTOS) have been carefully selected to have a friendly, flexible, and powerful experimental setup. Both must be simple to avoid discouraging those students with strong control system background but weak real-time system background when facing the programming part or vice versa to avoid discouraging those students with strong real-time system background but weak control system background when facing the controller design stage.

\section{A. Plant}

Many standard basic and advanced controller design methods rely on the accuracy of the plant mathematical model. The more accurate the model is, the more realistic the simulations are, and the better the observation of the effects of the controller on the plant is. Hence, the plant was selected among those for which an accurate mathematical model could easily be derived.

Plants such as an inverted pendulum or a direct current motor are the de facto plants for benchmark problems in control engineering [17]. However, their modeling is not trivial, and the resulting model is often not accurate. This leads to a first controller design that has to be adjusted by "engineering experience," thus requiring knowledge that is difficult to formalize and transmit to the students. To avoid such a kind of drawbacks, a simple electronic circuit in the form of $R C R C$ [Fig. 1(a)] was selected. The simplicity of its components and their simple and intuitive physical behavior have been the main reasons for its selection. Note however that experiments using other plants can be complementary to the approach presented here, e.g., [15] and [18]-[20]. Indeed, Lim [19] also proposes electronic circuits. However, they are slightly more sophisticated because they include operational amplifiers. Although richer dynamics can be achieved, the intuitive behavior and, thus, the modeling of operational amplifiers are not straightforward.

The selection of an electronic circuit as a plant has also another important advantage: Depending on the specific circuit, it can be directly plugged into a microcontroller without using 
intermediate electronic components, as shown in Fig. 1(b), where the zero-order hold (zoh) box represents the actuator and the box above represents the sampler, i.e., the transistor-transistor logic level signals provided by the microcontroller can be enough to carry out the control. Note that this is not the case, for example, for many mechanical systems. Such a simplification in terms of hardware reduces the modeling effort to study the plant, and no models for actuators or sensors are required. Additional benefits of these types of plants are that systems can be easily built, are cheap, have light weight, and can be easily transported and powered.

The control objective would be to have the circuit output voltage $V_{\text {out }}$ (controlled variable) track a reference signal or settle to a constant value while meeting, for example, the given transient response specifications, which mandates using tracking structures. The control will be achieved by sampling $V_{\text {out }}$, executing the control algorithm, and applying the calculated control signal to the circuit via varying the circuit input voltage $V_{\text {in }}$ (manipulated variable). Disturbances can be injected by a variable load voltage placed in parallel to the output voltage.

\section{B. Processing Platform}

The processing platform consists of the hardware platform and the RTOS. As a hardware platform, a microcontrollerbased architecture was selected because embedded systems are typically implemented using this type of hardware. Note, however, that too small microcontrollers may not be powerful enough for running an RTOS, as discussed in [22]. Among the several possibilities available on the market [23], it was decided to adopt the Flex board [24].

The Flex board (in its full version) represents a good compromise between cost, processing power, and programming flexibility. It was produced as a development board for building and testing real-time applications using standard components and open-source software. The board includes a Microchip dsPIC digital signal microcontroller dsPIC33FJ256MC710, a socket for the 100-pin plug-in module, an in-circuit debugger (ICD2) programmer connector, a Universal Serial Bus connector for direct programming, power supply connectors, a set of LEDs for monitoring the board, an onboard Microchip PIC18F2550 microcontroller for integrated programming, and a set of connectors for daughterboard piggybacking.

The board has several key benefits that make it suitable to be used for educational purposes. First, it has a robust electronic design, which is an important feature when it is employed by nonskilled users. Second, it has a modular architecture, which allows users to easily develop homemade daughterboards using standard components. A set of daughterboards can be added to the Flex board for easy development, such as a multibus board equipped with a controller area network (CAN), Ethernet, $\mathrm{I}^{2} \mathrm{C}$ (Inter-Integrated Circuit), and other communication protocols. On the one hand, the availability of CAN or Ethernet permits building and experimenting with networked control applications. On the other hand, the available networks can be used for debugging purposes or for extracting data from the board, which is a difficult task when dealing with embedded systems.
As far as the real-time kernel is concerned, different possibilities were considered. First, many well-known RTOSs, such as real-time Linux [25], target processors that may be too powerful for embedded applications, but more importantly, their internal structure is often too complex for those students with a low profile in (real-time) operating systems. Hence, it looks more desirable to work with small real-time kernels (e.g., [26]-[31] for small real-time kernels targeting small architectures) whose internals are accessible and easy to understand and modify, in order to tailor them to the specific application needs. On the other hand, from a user point of view, programming and configuring the kernel (including creating tasks, assigning priorities/periods/deadlines, and setting the scheduling policy) should be friendly enough to attract nonskilled programmers.

From the considerations mentioned previously, an Erika Enterprise real-time kernel [24] was selected. Erika provides full support to the Flex board in terms of drivers, libraries, programming facilities, and sample applications. The kernel, available under the General Public License and OSEK (Open Systems and their Interfaces for the Electronics in Motor Vehicles [32]) compliant, is an RTOS for small microcontrollers based on an API similar to those proposed by the OSEK consortium. The kernel gives support for preemptive and nonpreemptive multitasking and implements several scheduling algorithms [33]. The API provides support for tasks, events, alarms, resources, application modes, semaphores, and error handling. All these features permit enforcing real-time constraints to application tasks to show students the effects of sampling periods, delays, and jitter on control performance.

The development environment for Erika Enterprise is based on cross compilation, avoiding typical student misconceptions when the development platform and the target share the same hardware. A tool, named RT-Druid [24] (based on Eclipse [34]), can be used as a default development platform to program in C, with support from Microchip for the compiler and for the programming development kit. The latter is important because Microchip Web pages [35] are always a good place to share experiment experiences and code: a good place for instructors and students to visit. RT-Druid implements an OSEK Implementation Language (OIL) compiler, which is able to generate the kernel configuration from an OIL specification. Apart from programming in $\mathrm{C}$, the Flex board can also be programmed automatically using the Scilab/Scicos [36] code generator (similar to what can be done with MATLAB/Simulink [37] and its Real-Time Workshop, as used, for example, in [38] for rapid control prototyping). This is an important benefit for nonskilled C programmers.

From an education point of view, it is also important to note that there is a possibility to build a community around this processing platform to create a repository of control software for education. In fact, a set of application notes that describe a set of control experiments (inverted pendulum, ball and plate, etc.) developed with Erika on Flex can be found in [24].

Finally, it must be stressed that the price of the Flex board lies in the lower bound of evaluation board prices and that the Erika kernel and the associated development tools are open source, available for free, or available for free in student edition format. Hence, it is an economically attractive option. 


\section{EXAMPLE OF THE LABORATORY EXPERIMENT}

This section presents some of the activities in the form of problems and solutions (and observations) required to carry out the laboratory experience, which are later ordered in the work plan. The emphasis is in the control analysis and design part.

\section{A. Problem 1. Plant Modeling}

If $q_{i}$ represents the charge on capacitor $C_{i}$, the differential equations of the circuit, in terms of the currents $\dot{q}_{i}$ at each $R_{i}$, are given by

$$
\begin{aligned}
\dot{q}_{1} R_{1}+\left(q_{1}-q_{2}\right) \frac{1}{C_{1}} & =V_{\mathrm{in}} \\
\dot{q}_{2} R_{2}+\left(q_{2}-q_{1}\right) \frac{1}{C_{1}}+q_{2} \frac{1}{C_{2}} & =0 \\
q_{2} \frac{1}{C_{2}} & =V_{\text {out }}
\end{aligned}
$$

For example, using state-space formalism, a state-space form is given by

$$
\begin{aligned}
\dot{x}(t)= & {\left[\begin{array}{cc}
0 & 1 \\
\frac{-1}{R_{1} R_{2} C_{1} C_{2}} & -\frac{R_{1} C_{1}+R_{2} C_{2}+R_{1} C_{2}}{R_{1} R_{2} C_{1} C_{2}}
\end{array}\right] x(t) } \\
& +\left[\begin{array}{c}
0 \\
\frac{1}{R_{1} R_{2} C_{1} C_{2}}
\end{array}\right] u(t) \\
y(t)= & {\left[\begin{array}{ll}
1 & 0
\end{array}\right] x(t) }
\end{aligned}
$$

where $u(t)$ is the control signal, $y(t)$ is the plant output, and $x(t)=\left[\begin{array}{ll}x_{1} & x_{2}\end{array}\right]$ is the state vector, where $x_{1}$ corresponds to the output voltage $V_{\text {out }}$ and $x_{2}$ is $\dot{q}_{2} / C_{2}$.

Observation 1: The modeling of the plant could have been also done in terms of a transfer function (see [39] for the analysis). Even obtaining the differential equations is a good exercise. Adopting the state-space formalism may add another benefit if using (2). Since only the output voltage $x_{1}$ can be physically measured, the control algorithm requires the use of observers for predicting $x_{2}$. This opens the door to experimenting with several types of observers, and the implementation of the controller has to include them. Furthermore, the selection of the state variables is arbitrary, and therefore, students have to take design decisions. For example, the voltages in both capacitors could also have been chosen as state variables. In any case, if possible, it is interesting to choose the state variables in such a way that the controlled variable is directly available through the output matrix in order to minimize computations in the microcontroller.

\section{B. Problem 2. Electronic Components}

The selection of the electronic components is very important for several reasons. The output impedance must be low enough to properly connect the circuit to the analog-to-digital converter (ADC) or to some external instrumentation, such as an oscilloscope. For example, given the initial components $R_{1}=R_{2}=1 \mathrm{k} \Omega$ and $C_{1}=C_{2}=33 \mu \mathrm{F}$, a manageable circuit impedance is obtained. With these components, the state-space model becomes

$$
\begin{aligned}
& \dot{x}(t)=\left[\begin{array}{cc}
0 & 1 \\
-976.56 & -93.75
\end{array}\right] x(t)+\left[\begin{array}{c}
0 \\
976.56
\end{array}\right] u(t) \\
& y(t)=\left[\begin{array}{ll}
1 & 0
\end{array}\right] x(t) .
\end{aligned}
$$

Observation 2: Students can be given other values. For example, with $R_{1}=R_{2}=330 \mathrm{k} \Omega$ and $C_{1}=C_{2}=100 \mathrm{nF}$, it is easy to see that the equivalent output impedance is too high for the ADC. To derive such a conclusion, students have to consult the dsPIC datasheet.

\section{Problem 3. Open-Loop Simulation}

Open-loop dynamics can be observed by injecting reference signals to the circuit via its input $V_{\text {in }}$. For example, by injecting a square wave that oscillates between 1 and $2.5 \mathrm{~V}$ at $1 \mathrm{~Hz}$, the obtained dynamics are shown in Fig. 2(a). The voltage output $V_{\text {out }}$ (solid curve) slowly tracks the reference (dashed curve).

Observation 3: The electronic components determine the circuit open-loop dynamics. Students must be aware of this by playing with different electronic components. In addition, simulation can be done by standard software packages used in control engineering (e.g., MATLAB/Simulink and Scilab/Scicos) or by programming the response in $\mathrm{C}$ or even using a spreadsheet application.

\section{Problem 4. Controller Design: Sampling Period and Performance Specifications}

For the chosen plant, there can be different control goals. A possibility can be to modify the $R C R C$ transient response by accelerating it or by arbitrarily achieving a given overshoot. In any case, the selection of the sampling period and the controller itself have a strong impact on the control performance. Furthermore, they have to be selected and designed taking into account the processing platform. If avoiding intermediate electronics between the plant and dsPIC is the assumption, the control signal $\left(V_{\text {in }}\right)$ can be generated using pulsewidth modulation [(PWM); by adjusting the duty cycle], and the controlled variable $\left(V_{\text {out }}\right)$ can be obtained through the ADC. Therefore, the reference, the sampling period, and the controller must be chosen/designed so that the voltage levels of the control signal and the generated peak current levels lie within the hardware limitations.

Given the previous square reference signal, through an iterative design stage, and according to standard rules of thumb, two state feedback controllers can be designed: one that accelerates the response, named fast controller, and another that produces overshoot, named overshoot controller. For the fast controller, the period is set to $h=0.01 \mathrm{~s}$, and the discrete state feedback controller is designed to place the continuous closed-loop poles at $p_{1,2}=-30$ (note that the open-loop system has poles at -12 and -81 approximately). The overshoot controller has a sampling period of $h=0.1 \mathrm{~s}$, and the controller places the closed-loop poles at $p_{1,2}=-10 \pm 20 i$. 


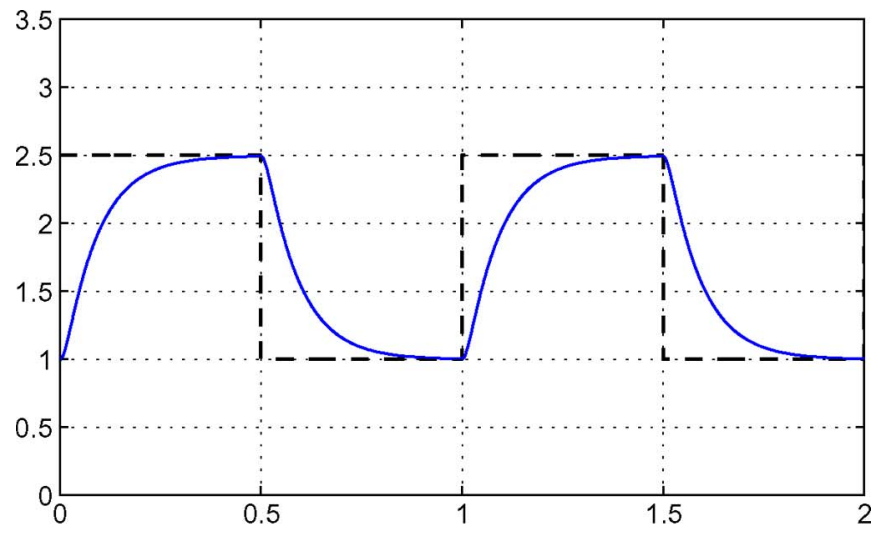

(a)

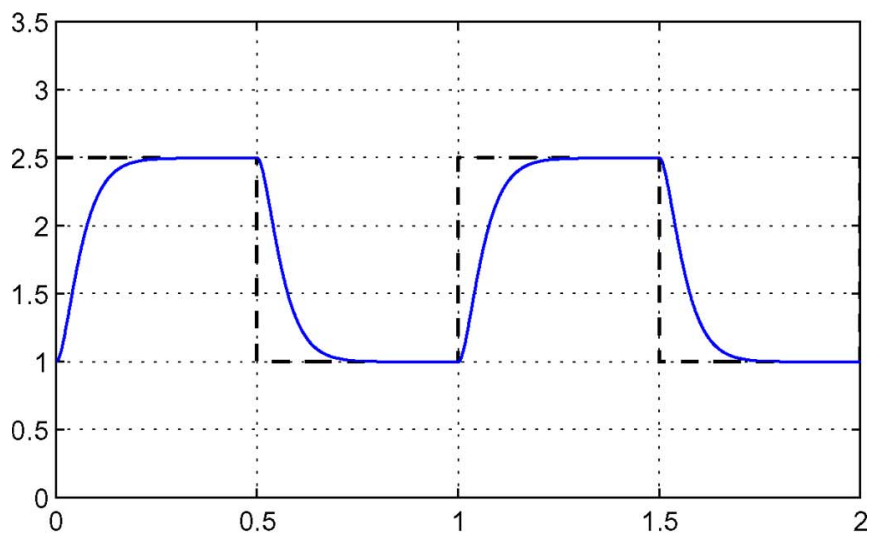

(b)

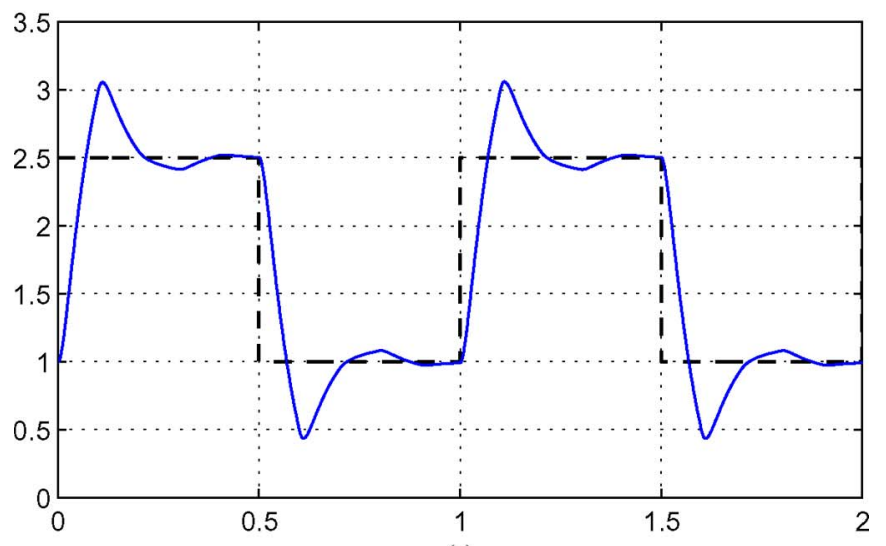

(c)

Fig. 2. Simulated $R C R C$ responses. (a) Open-loop response. (b) Faster closed-loop response. (c) Overshoot closed-loop response.

It is worth noting that, using only standard rules of thumb for selecting sampling periods [39], both controllers should have a slightly shorter sampling period. The fast controller should be given a period of $h=0.007 \mathrm{~s}$ and the overshoot controller a period of $h=0.03 \mathrm{~s}$. However, since longer sampling periods result in lower controller resource demands, an iterative simulation process was used to find longer sampling periods for both controllers without introducing unacceptable performance degradation.

Observation 4: Selecting sampling periods, desired closedloop poles, or even having a higher amplitude for the reference signal may cause the values of the control signal to be out of range. Relations illustrating tradeoffs in the design of embedded control systems can also be taught to the students, such as showing that increasing sampling rates mean stronger control signals (saturation problem) but also more processor usage (feasibility/schedulability problem).

\section{E. Problem 5. Controller Design: Tracking}

The controller design has to consider that the goal is to track a voltage. The standard tracking structure [39], that includes $N_{u}$ as the matrix for the feedforward signal to eliminate steadystate errors and $N_{x}$ as the matrix that transforms the reference $r$ into a reference state, can be adopted. Following the case study, $N_{u}=[1]$ and $N_{x}=\left[\begin{array}{ll}1 & 0\end{array}\right]$, and the discrete gains are $K=[0.0685-0.0249]$ or $K=\left[\begin{array}{ll}1.0691 & -0.0189\end{array}\right]$ for the fast or overshoot controller, respectively.

Observation 5: Students can practice other tracking structures, such as integral control, and can study whether the code of the controller would suffer significant changes.

\section{F. Problem 6. Controller Design: Closed-Loop Simulation}

The simulated closed-loop response for the fast and overshoot controllers is shown in Fig. 2(b) and (c), respectively.

Observation 6: As before, simulations can be done using different methods.

\section{G. Problem 7. Controller Design: Observers}

For the simulation, the two state variables are available. However, in the real experiment, an observer must be included. For simplicity in coding the control task, a reduced observer can be chosen for observing the second state variable $x_{2}$. For example, the observer discrete gain is $K_{r}=-37.81$ or $K_{r}=-13.42$ for the fast or overshoot controller if the observer continuous closed-loop pole is located at $p_{o b}=-50$.

Observation 7: Students can design and evaluate by simulating different types of observers (reduced, complete, etc.) with different dynamics, i.e., they can also evaluate the effect of different locations for the observer poles. From an implementation point of view, students can also assess the effect that splitting the control algorithm into two parts (calculate control signal and update state) has on input-output delays and schedulability [41].

\section{H. Problem 8. Implementation: Kernel Configuration and Control Algorithm}

The first implementation involves coding the controller in a periodic task that will execute in isolation on top of Erika. The main pseudocodes are shown in Figs. 3-5.

Fig. 3 shows the conf.oil file that specifies the kernel configuration with the Earliest Deadline First (EDF [40]) scheduling algorithm and a periodic task that will be used to implement, for instance, the fast controller. The basics of the main code are shown in Fig. 4. First, the timer T1 is initialized and, together with SetRelAlarm, will produce the periodic activation of the control task every $10 \mathrm{~ms}$ (the processor speed was configured at 40 million instructions/s). Fig. 5 shows the control task code, including the observer and the tracking structure. 


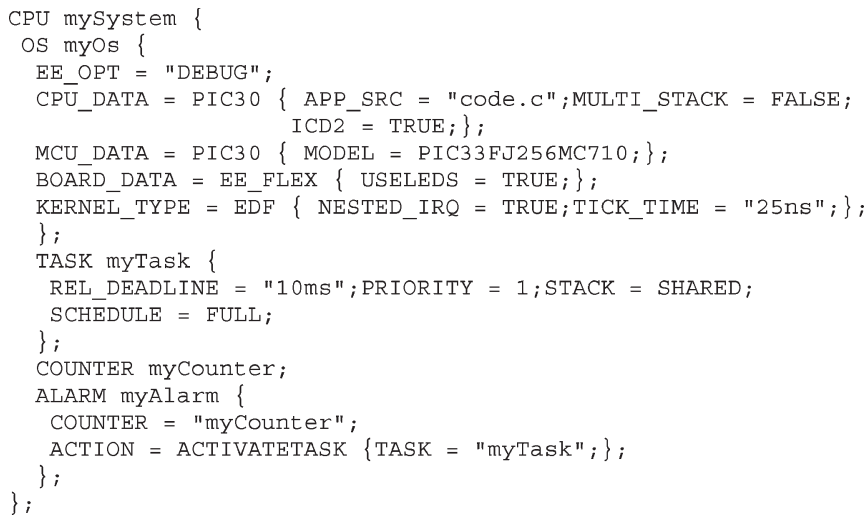

Fig. 3. Kernel configuration file.

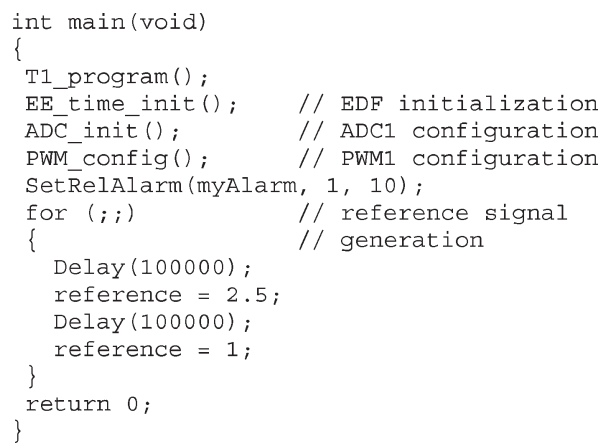

Fig. 4. Main code.

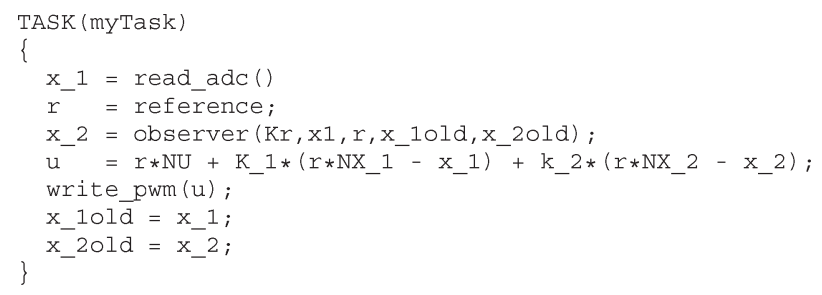

Fig. 5. Control task code.

Observation 8: Carrying out the kernel configuration and programming the controller could be taught following an ordered sequence of steps, such as the following: 1) introduction to kernel configuration; 2) introduction to periodic tasks; and 3 ) introduction to input/output operations (PWM, ADC).

\section{Problem 9. Implementation: Setup and Monitoring Details}

Fig. 6(a) shows the experimental setup that includes an oscilloscope (for displaying the circuit output voltage) to show the open-loop response [Fig. 6(b)] and the closed-loop responses [Fig. 7(a) and (b)] achieved by each controller executing in isolation. The oscilloscope screenshots confirm that the implementation achieves the control goal: The system output performs the desired fast tracking or achieves the specified overshoot.

Observation 9: An oscilloscope has been used to monitor both the responses. It can also be of interest to monitor whether the control task executes when specified. Another option could be to use the Multibus board to send the data of interest via Ethernet or any other available communication protocol. This

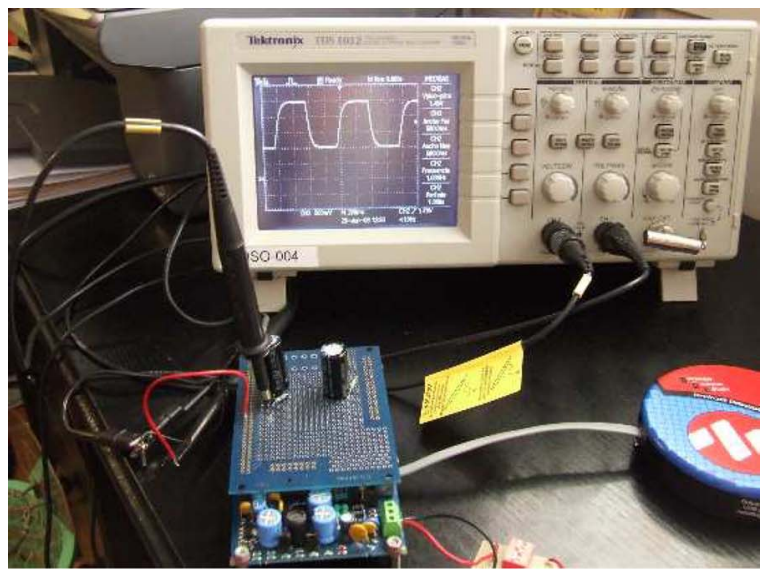

(a)

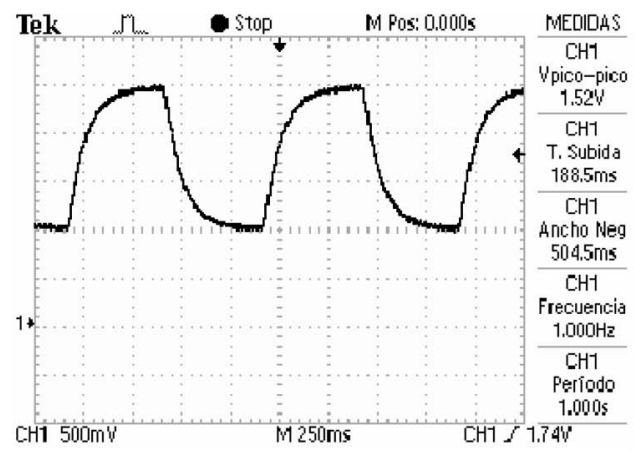

(b)

Fig. 6. Experiment setup and monitoring. (a) Setup. (b) Open-loop response.

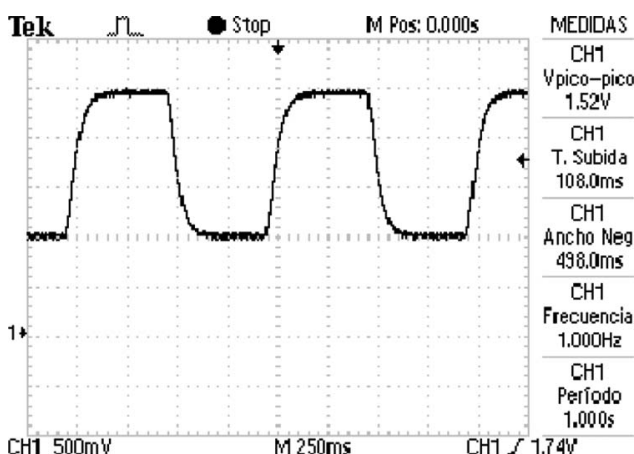

(a)

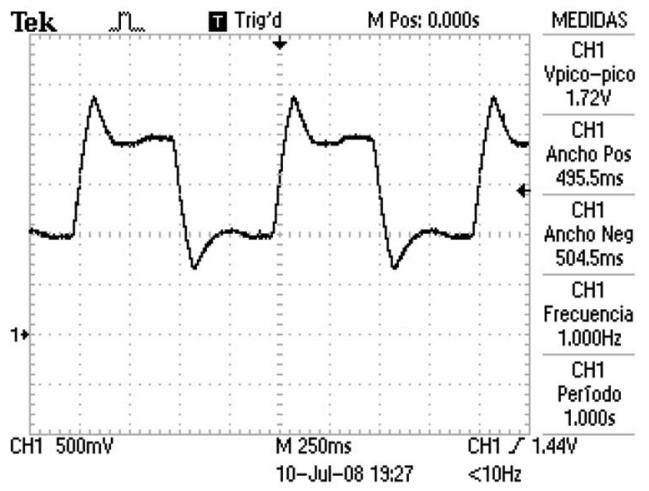

(b)

Fig. 7. $R C R C$ responses. (a) Fast closed-loop response. (b) Overshoot closed-loop response. 
would pose interesting challenges in terms of the real-time system such as noninvasive debugging.

\section{J. Problem 10. Multitasking: Simulation}

A second implementation is introduced to illustrate more advanced concepts. In the previous implementation, a control task was executing in isolation. However, in many high-technology systems, the processor is used not only for the control computation but also for interrupt handling, error management, monitoring, etc. Furthermore, it is known that, in multitasking real-time control systems, jitters, i.e., timing interferences on control tasks due to the concurrent execution of other tasks, deteriorate the control loop performance [41]. The objective of this implementation is to observe these degrading effects and implement corrective actions, e.g., [42]-[44].

A starting point is to inject a new task in the kernel for each control task. The new task, named noisy task, when executed together with the fast controller, is given a period (and relative deadline) of $11 \mathrm{~ms}$, and it imposed an artificial execution time of $9 \mathrm{~ms}$. The noisy task that goes together with the overshoot controller has a period and relative deadline of $110 \mathrm{~ms}$ with $90 \mathrm{~ms}$ of execution time. The simulation of each multitasking system was done in the TrueTime simulator [45]. Putting together each control task with the corresponding noisy task under EDF results in timing variability (jitter) for the control task, as shown for the first multitasking system in the schedule in Fig. 8(a). In this figure, the bottom curve represents the execution of the fast controller task, whereas the top curve represents the execution of the noisy task. In each graph, the low-level line denotes no execution (i.e., intervals in which the processor is idle), the middle-level line denotes a task ready to execute (i.e., waiting in the ready queue), and the high-level line denotes a task in execution. Note that the control task has a measured execution time of $0.12 \mathrm{~ms}$, which is much less than that in the noisy task.

Looking at the plant responses in Fig. 8(b) and (c), it can be appreciated that the fast controller does not exhibit control performance degradation, while the overshoot controller suffers some degradation: Overshoots are bigger, square amplitude differs, transient response varies, etc. This fact indicates that the current control design for the fast controller is robust against jitters induced by scheduling, while the second one is more fragile.

Observation 10: Adopting an iterative simulation study, students can learn which parameters play an important role when jitters appear. Are shorter sampling periods, or nonovershooted responses, a guarantee for having robust control designs? Which role do deadlines play in reducing jitters? For further questions and solutions, see [46] and references therein.

\section{K. Problem 11. Multitasking: Implementation and Monitoring Details}

The new implementation requires specifying the noisy task by modifying the kernel oil file in terms of defining the new task and the associated alarm. Furthermore, the new task has to be coded: Forcing an artificial execution time is achieved by placing a delay into the code. The main code has to be modified to configure the new alarm associated to the new task.

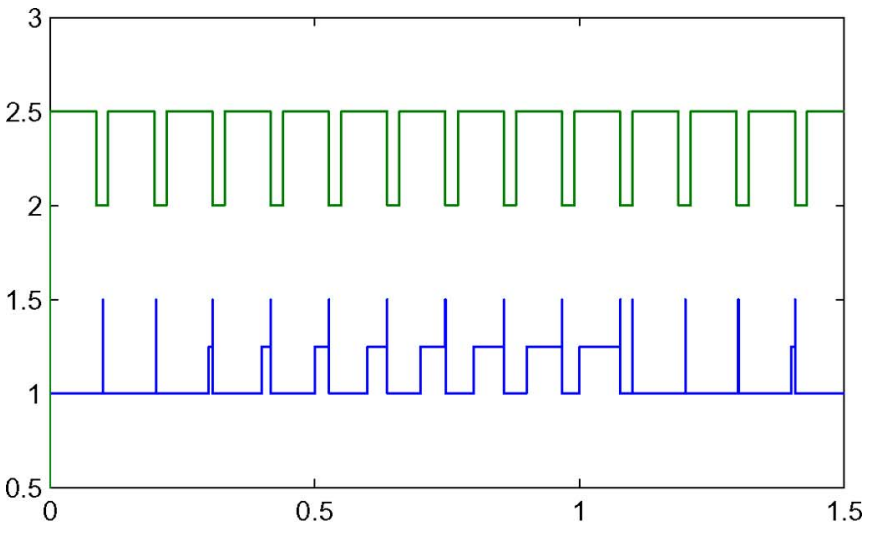

(a)

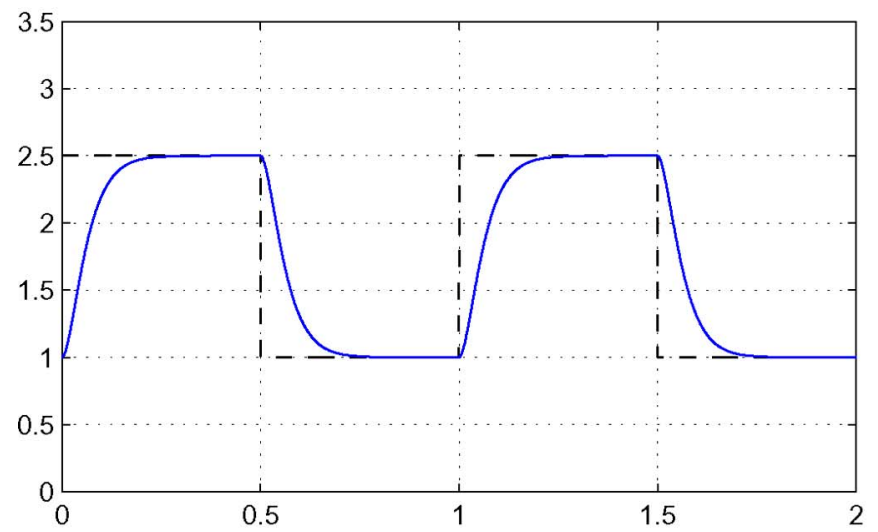

(b)

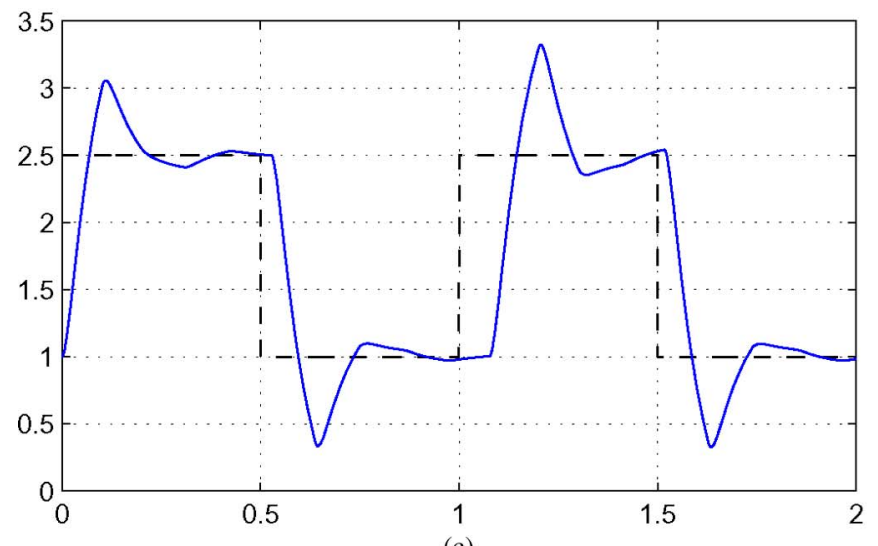

(c)

Fig. 8. Simulated multitasking $R C R C$ responses and schedule. (a) Partial schedule. (b) Faster multitasking closed-loop response. (c) Overshoot multitasking closed-loop response.

After the implementation, in the first multitasking system, it can be verified that scheduling conflicts [as illustrated in the simulated schedule shown in Fig. 8(a)] may occur, as shown in Fig. 9(a). In this figure, the execution of the fast controller task sets an output pin to 0 and 1 at each job start and finishing time, and the noisy task sets an output pin to 0 and 0.5 at each job start and finishing time. In addition, similar responses for the fast and overshoot controllers are obtained [see Fig. 9(b) and (c)], showing that the overshoot controller suffers degradation from jitters, while the fast controller shows the same response as it is executed in isolation. For illustrative purposes, Fig. 10(a) shows the overshoot controller response when executing in 


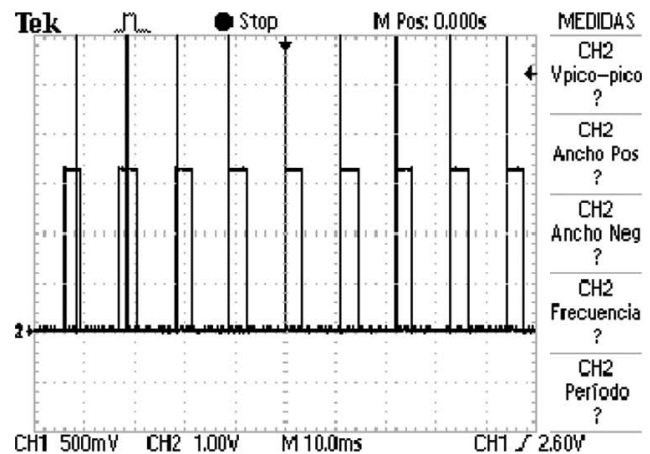

(a)

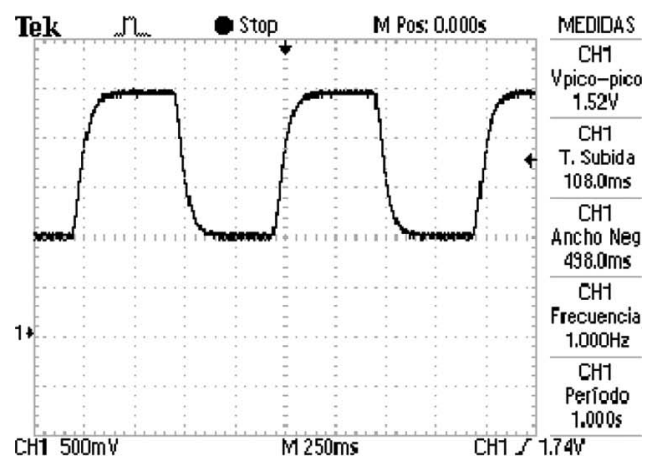

(b)

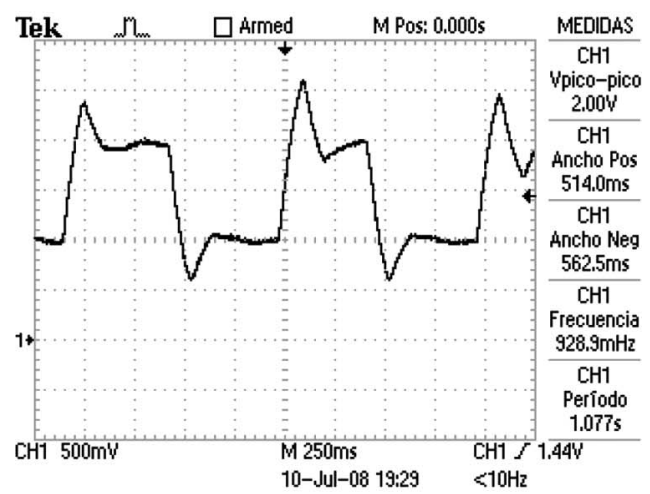

(c)

Fig. 9. Implemented multitasking $R C R C$ responses and schedule. (a) Partial schedule. (b) Faster multitasking response. (c) Overshoot multitasking response.

isolation (dark curve) and when executing in the multitasking system (gray curve).

Observation 11: Undergraduate students may work the experiment up to this problem. It shows the importance of concurrence and resource sharing with respect to control performance in a multitasking embedded control system.

\section{Problem 12: Multitasking: Design for Eliminating or Minimizing the Jitter Problem}

Recent research literature has faced the problems introduced by jitter, and many solutions have been proposed. Here, the solution proposed by Lozoya et al. [44] has been adopted.

The basic idea is to synchronize the operations within each control loop at the actuation instants. In this way, the time elapsed between consecutive actuation instants, named $t_{k-1}$ and $t_{k}$, is exactly equal to the sampling period $h$. Within this time interval, the system state is sampled, named $x_{s, k}$, and

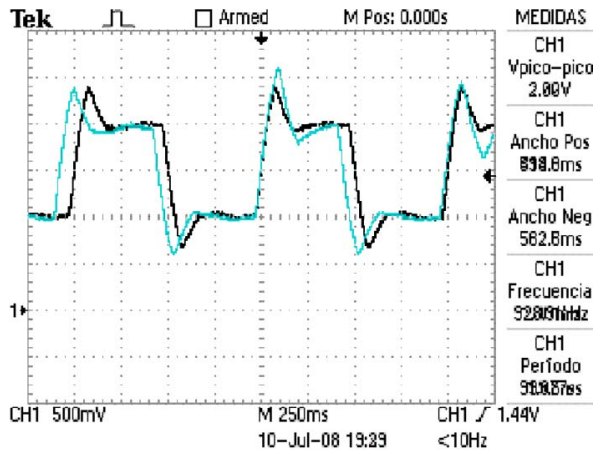

(a)

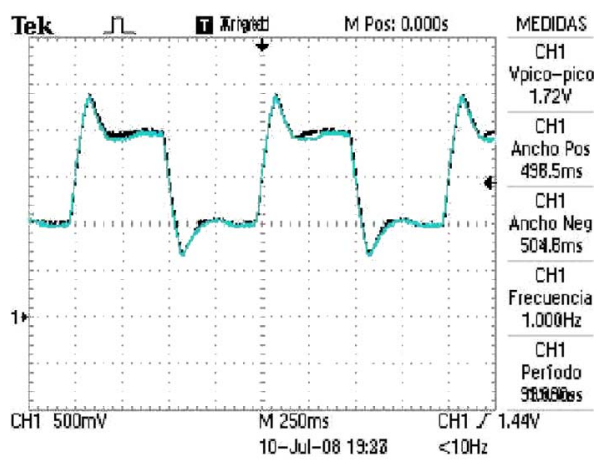

(b)

Fig. 10. Overshoot controller: Degradation and solution. (a) Overshoot controller with/without jitters. (b) Overshoot controller eliminating the jitter problem.

the sampling time is recorded $t_{s, k} \in\left(t_{k-1}, t_{k}\right)$. The difference between this time and the next actuation time

$$
\tau_{k}=t_{k}-t_{s, k}
$$

is used to estimate the state at the actuation instant as

$$
\hat{x}_{k}=\Phi\left(\tau_{k}\right) x_{s, k}+\Gamma\left(\tau_{k}\right) u_{k-1}
$$

where $\Phi(t)=e^{A t}, \Gamma(t)=\int_{0}^{t} e^{A s} \mathrm{dsB}$, with $A$ and $B$ being the system and input matrices in (3), and $u_{k-1}$ is the previous control signal. Then, making use of $\hat{x}_{k}$, the control command is computed using the original control gain $K$ as

$$
u_{k}=K \hat{x}_{k} .
$$

The control command $u_{k}$ is held until the next actuation instant. A control strategy using (4)-(6) relies on the time reference given by the actuation instants, if $u_{k}$ is applied to the plant by hardware interrupts, for example. In addition, samples are not required to be periodic because $\tau_{k}$ in (4) can vary at each closed-loop operation.

After implementing this strategy on the overshoot controller in the multitasking system, Fig. 10(b) shows the result. Specifically, it shows the overshoot controller response when executing in isolation (dark curve) and when executing in the multitasking system using the algorithm that eliminates jitters (gray curve).

Observation 12: The problem presented previously and its solution can be split into several tasks, like analysis and modeling of the new control algorithm, implementation of the control algorithm, etc. An interesting issue is how synchronized 
actuation instants can be forced in the kernel. For example, a solution could be to use a periodic task for computing $u_{k}$ and another periodic task for applying $u_{k}$ at the required time. Another solution could be to enforce synchronized executions at the kernel level, using the EDF tick counter. Moreover, since different solutions to the jitter problem, such as that in [42] or [43], could have also been applied, students that are more confident or interested in specific fields can select the solution that better meets their preferences.

\section{Tentative Work Plan and Its Application TO A SPECIFIC COURSE}

The previous section has detailed some of the steps required to successfully carry out the laboratory activity presented in this paper. This section summarizes them in order to propose a tentative work plan that is divided into several sessions, with each one being a 2-h laboratory.

1) S1-Introduction: Introduction to the activity and simulation of the open-loop response after obtaining the state-space form of the $R C R C$ circuit from the circuit differential equations (1) (consider random values for $R$ and $C$ ). Here, it is assumed that a state-space notation is chosen.

2) S2-Problem specification (a): This session should be used to specify the problem in terms of the levels for the reference signal and for the discrete controller design, which includes selecting the sampling period and the closed-loop pole locations, if pole placement is used. Other control approaches, like optimal control, could also be used.

3) S3-Problem specification (b): To complement the previous session, observers should also be designed and simulated. The outcome of this session should be the complete simulation setup.

4) S4-Basic implementation (a): Build the $R C R C$ circuit and verify its dynamics in open loop. Start the controller implementation in a periodic hard real-time task in the processing platform.

5) S5-Basic implementation (b): Finish the controller implementation and test its correctness.

6) S6-Multitasking (a): Incorporate a noisy task in the simulation setup to evaluate the effects of jitter. This step would require using, for example, the TrueTime simulator.

7) S7-Multitasking (b): Incorporate the noisy task in the implementation and validate the previous simulation results.

8) S8-Advanced implementation (a): If degradation in control performance is detected in the previous session, simulate advanced control algorithms or adopt real-time techniques to solve or reduce the jitter problem.

9) S9-Advanced implementation (b): Implement the previous solutions and validate them.

Note that the program timing, the layout, and the set of covered topics should be adapted to the particular needs/ background of the target audience or to the goals of a specific curriculum. For example, the proposed activity has been introduced as part of the curriculum of the two-year master degree on Automatic Control and Industrial Electronics in the School of Engineering of Vilanova i la Geltrú (EPSEVG) of the Technical University of Catalonia (UPC) [47]. In particular, since 2007, the experiment has been tailored to become part of the laboratory for the Control Engineering course, which covers continuous and discrete linear time-invariant (LTI) control systems, as well as nonlinear control systems, all using state-space formalism. Sessions S1-S5 were adopted for the laboratory of the discrete LTI control system part.

The Control Engineering course can be followed by students either in the first semester of the first year or in the first semester of the second year. Students choosing the second option simultaneously attend a course on real-time systems. Therefore, within the same classroom, not all the students are familiar with real-time systems. To overcome this apparent drawback, teams of three students were formed containing at least a student with competence on real-time systems. Within such heterogeneous teams in terms of skills and theoretical background, it was observed that students took their responsibilities, and teamwork was significantly improved.

As in every course edition, after finishing the discrete LTI control system part, a short and simple questionnaire is given to the students to let them evaluate several aspects of this part of the course. The question Do the laboratory activities permit to better understand the theoretical concepts? is the only one related to the laboratories. Looking at the students' answers and taking into account that, before introducing the presented experiment, the laboratory activities were focused on the simulation of an inverted pendulum, the percentage of students appreciating the practical part has increased significantly. Although the standard course evaluation indicates this positive trend, a more complete evaluation tailored to the introduction of this experiment is required.

\section{CONCLUSION}

This paper has presented a laboratory activity to be integrated in the education curriculum of embedded control system engineers. The activity consists of a real-time controller of an $R C R C$ electronic circuit. The potential benefits, the competences to be acquired, and the expected learning outcomes for students have been presented. The selection of the plant and processing platform has been discussed. Extensive details of a sample implementation have been presented, and a tentative work plan for carrying out the activity has been provided.

In summary, the proposed activity poses several real challenges to the students that can be met by putting together interdisciplinary skills (electronics, real-time systems, control theory, and programming) toward a single goal: building a working system.

\section{REFERENCES}

[1] D. J. Jackson and P. Caspi, "Embedded systems education: Future directions, initiatives, and cooperation," SIGBED Rev., vol. 2, no. 4, pp. 1-4, Oct. 2005.

[2] A. Crespo, J. Vila, F. Blanes, and I. Ripoll, "Real-time education in a control engineering curriculum," in Proc. 3rd IEEE Real-Time Syst. Educ. Workshop, 1998, pp. 112-116. 
[3] K. G. Ricks, D. J. Jackson, and W. A. Stapleton, "Incorporating embedded programming skills into an ECE curriculum," SIGBED Rev., vol. 4, no. 1, pp. 17-26, Jan. 2007.

[4] W. A. Halang, "A curriculum for real-time computer and control systems engineering," IEEE Trans. Educ., vol. 33, no. 2, pp. 171-178, May 1990.

[5] P. Caspi, A. Sangiovanni-Vincentelli, L. Almeida, A. Benveniste, B. Bouyssounouse, G. Buttazzo, I. Crnkovic, W. Damm, J. Engblom, G. Folher, M. Garcia-Valls, H. Kopetz, Y. Lakhnech, F. Laroussinie, L. Lavagno, G. Lipari, F. Maraninchi, P. Peti, J. de la Puente, N. Scaife, J. Sifakis, R. de Simone, M. Torngren, P. Veríssimo, A. J. Wellings, R. Wilhelm, T. Willemse, and W. Yi, "Guidelines for a graduate curriculum on embedded software and systems," ACM Trans. Embed. Comput. Syst., vol. 4, no. 3, pp. 587-611, Aug. 2005.

[6] A. Sangiovanni-Vincentelli and A. Pinto, "An overview of embedded system design education at Berkeley," ACM Trans. Embed. Comput. Syst., vol. 4, no. 3, pp. 472-499, Aug. 2005.

[7] K. G. Ricks, D. J. Jackson, and W. A. Stapleton, "An embedded systems curriculum based on the IEEE/ACM model curriculum," IEEE Trans. Educ., vol. 51, no. 2, pp. 262-270, May 2008.

[8] D. Davcev, B. Stojkoska, S. Kalajdziski, and K. Trivodaliev, "Project based learning of embedded systems," in Proc. 2nd WSEAS Int. Conf. Circuits, Syst., Signal Telecommun., 2008, pp. 120-125.

[9] S. Nooshabadi and J. Garside, "Modernization of teaching in embedded systems design-An international collaborative project," IEEE Trans. Educ., vol. 49, no. 2, pp. 254-262, May 2006.

[10] J. W. Bruce, J. C. Harden, and R. B. Reese, "Cooperative and progressive design experience for embedded systems," IEEE Trans. Educ., vol. 47, no. 1, pp. 83-92, Feb. 2004.

[11] J. Fernandez, R. Marin, and R. Wirz, "Online competitions: An open space to improve the learning process," IEEE Trans. Ind. Electron., vol. 54, no. 6, pp. 3086-3093, Dec. 2007.

[12] U. Munz, P. Schumm, A. Wiesebrock, and F. Allgower, "Motivation and learning progress through educational games," IEEE Trans. Ind. Electron., vol. 54, no. 6, pp. 3141-3144, Dec. 2007.

[13] L. Gomes and S. Bogosyan, "Current trends in remote laboratories," IEEE Trans. Ind. Electron., vol. 56, no. 12, pp. 4744-4756, Dec. 2009.

[14] D. T. Rover, R. A. Mercado, Z. Zhang, M. C. Shelley, and D. S. Helvick, "Reflections on teaching and learning in an advanced undergraduate course in embedded systems," IEEE Trans. Educ., vol. 51, no. 3, pp. 400412, Aug. 2008.

[15] K.-E. Årzén, A. Blomdell, and B. Wittenmark, "Laboratories and realtime computing: Integrating experiments into control courses," IEEE Control Syst. Mag., vol. 25, no. 1, pp. 30-34, Feb. 2005.

[16] G. Buttazzo, "Research trends in real-time computing for embedded systems," ACM SIGBED Rev., vol. 3, no. 3, pp. 1-10, Jul. 2006.

[17] P. Horacek, "Laboratory experiments for control theory courses: A survey," Annu. Rev. Control, vol. 24, pp. 151-162, 2000.

[18] M. Moallem, "A laboratory testbed for embedded computer control," IEEE Trans. Educ., vol. 47, no. 3, pp. 340-347, Aug. 2004.

[19] D.-J. Lim, "A laboratory course in real-time software for the control of dynamic systems," IEEE Trans. Educ., vol. 49, no. 3, pp. 346-354, Aug. 2006.

[20] M. Huba and M. Simunek, "Modular approach to teaching PID control," IEEE Trans. Ind. Electron., vol. 54, no. 6, pp. 3112-3121, Dec. 2007.

[21] VxWorks Operating System from WindRiver. [Online]. Available: http://www.windriver.com/

[22] R. Marau, P. Leite, M. Velasco, P. Martí, L. Almeida, P. Pedreiras, and J. M. Fuertes, "Performing flexible control on low cost microcontrollers using a minimal real-time kernel," IEEE Trans. Ind. Informat., vol. 4, no. 2, pp. 125-133, May 2008.

[23] F. Salewski, D. Wilking, and S. Kowalewski, "Diverse hardware platforms in embedded systems lab courses: A way to teach the differences," SIGBED Rev., vol. 2, no. 4, pp. 70-74, Oct. 2005.

[24] Evidence srl. [Online]. Available: http://www.evidence.eu.com/

[25] Real-time Linux Found., Inc. [Online]. Available: http://www. realtimelinuxfoundation.org/

[26] J. A. Stankovic and K. Ramamritham, "The spring kernel: A new paradigm for real-time operating systems," SIGOPS Oper. Syst. Rev., vol. 23, no. 3, pp. 54-71, Jul. 1989.

[27] K. M. Zuberi, P. Pillai, and K. G. Shin, "Emeralds: A small-memory realtime microkernel," in Proc. 7th ACM Symp. Oper. Syst. Principles, 1999, pp. 277-299.

[28] P. Gai, G. Lipari, L. Abeni, M. di Natale, and E. Bini, "Architecture for a portable open source real-time kernel environment," in Proc. 2nd Real-Time Linux Workshop/Hand's On Real-Time Linux Tu- torial, Nov. 2000, 9 pages. [Online]. Available: http://www.osadl.org/ RTLWS-2000.rtlws-2000.0.html

[29] E. Mumolo, M. Nolich, and M. Noser, "A hard real-time kernel for Motorola microcontrollers," in Proc. 23rd Int. Conf. Inf. Technol. Interfaces, Jun. 2001, pp. 75-80.

[30] P. Gai, L. Abeni, M. Giorgi, and G. Buttazzo, "A new kernel approach for modular real-time systems development," in Proc. 13th IEEE Euromicro Conf. Real-Time Syst., Jun. 2001, pp. 199-206.

[31] D. Henriksson and A. Cervin, "Multirate feedback control using the TinyRealTime kernel," in Proc. 19th Int. Symp. Comput. Inf. Sci., Antalya, Turkey, Oct. 2004, pp. 855-865.

[32] OSEK/VDX Portal. [Online]. Available: http://www.osek-vdx.org/

[33] G. Buttanzo, Hard Real-Time Computing Systems: Predictable Scheduling Algorithms and Applications. Norwell, MA: Kluwer, 1997.

[34] Eclipse Portal. [Online]. Available: http://www.eclipse.org/

[35] Microchip Portal. [Online]. Available: http://www.microchip.com/

[36] Scilab/Scicos Portal. [Online]. Available: http://www.scilab.org/

[37] MathWorks Portal. [Online]. Available: http://www.mathworks.com/

[38] D. Hercog, B. Gergic, S. Uran, and K. Jezernik, "A DSP-based remote control laboratory," IEEE Trans. Ind. Electron., vol. 54, no. 6, pp. $3057-$ 3068, Dec. 2007.

[39] K. Ogata, Modern Control Engineering, 4th ed. Englewood Cliffs, NJ: Prentice-Hall, 2001.

[40] C. L. Liu and J. W. Layland, "Scheduling algorithms for multiprogramming in a hard-real-time environment," J. Assoc. Comput. Machinery, vol. 20, no. 1, pp. 46-61, Jan. 1973.

[41] K.-E. Årzén, A. Cervin, J. Eker, and L. Sha, "An introduction to control and scheduling co-design," in Proc. 39th IEEE Conf. Decision Control, 2000, pp. 4865-4870.

[42] J. Skaf and S. Boyd, "Analysis and synthesis of state-feedback controllers with timing jitter," IEEE Trans. Autom. Control, vol. 54, no. 3, pp. $652-$ 657, Mar. 2009.

[43] P. Balbastre, I. Ripoll, J. Vidal, and A. Crespo, "A task model to reduce control delays," Real-Time Syst., vol. 27, no. 3, pp. 215-236, Sep. 2004.

[44] C. Lozoya, M. Velasco, and P. Martí, "The one-shot task model for robust real-time embedded control systems," IEEE Trans. Ind. Informat., vol. 4, no. 3, pp. 164-174, Aug. 2008.

[45] D. Henriksson, A. Cervin, and K.-E. Årzén, "TrueTime: Simulation of control loops under shared computer resources," in Proc. 15th IFACWorld Congr., 2002, 7 pages. [Online]. Available: http://www. ifac-papersonline.net/Detailed/26606.html

[46] Y. Wu, E. Bini, and G. Buttazzo, "A framework for designing embedded real-time controllers," in Proc. 14th IEEE Int. Conf. Embed. Real-Time Comput. Syst. Appl., Aug. 2008, pp. 303-311.

[47] EPSEVG School Portal. [Online]. Available: http://www.epsevg.upc.edu/

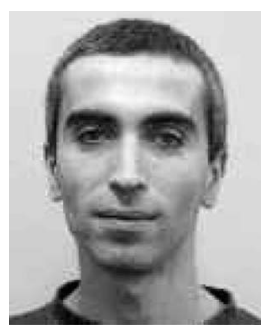

Pau Martí (M’02) received the B.S. degree in computer science and the Ph.D. degree in automatic control from the Technical University of Catalonia (UPC), Barcelona, Spain, in 1996 and 2002, respectively.

Since 1996, he has been an Assistant Professor with the Department of Automatic Control, UPC. During his Ph.D., he was a Visiting Student at Mälardalen University, Västerås, Sweden. From 2003 to 2004, he was a Research Fellow with the Computer Science Department, University of California, Santa Cruz. His research interests include embedded systems, control systems, real-time systems, and communication systems.

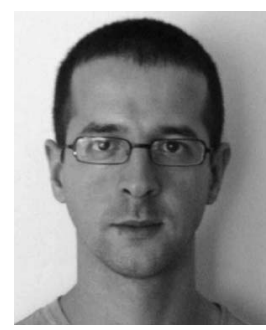

Manel Velasco received the B.S. degree in maritime engineering and the Ph.D. degree in automatic control from the Technical University of Catalonia (UPC), Barcelona, Spain, in 1999 and 2006, respectively.

Since 2002, he has been an Assistant Professor with the Department of Automatic Control, UPC. His research interests include artificial intelligence, real-time control systems, and collaborative control systems, particularly on redundant controllers and multiple controllers with self-interacting systems. 


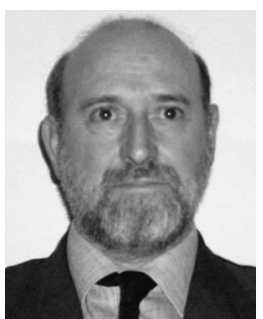

Josep M. Fuertes (S'74-M'96) received the B.S. degree in industrial engineering and the Ph.D. degree from the Technical University of Catalonia (UPC), Barcelona, Spain, in 1976 and 1986, respectively.

From 1975 to 1986, he was a Researcher with the Institut de Cibernética (Spanish Consejo Superior de Investigaciones Científicas). In 1987, he became a Permanent Professor with UPC. In 1987, he got a position for a year at the Ernest Orlando Lawrence Berkeley National Laboratory, Berkeley, CA, where he was a Visiting Scientific Fellow for the design of the Active Control System of the W. M. Keck 10-m segmented telescope (Hawaii). In 1992, he was responsible of the University Research Line in Advanced Control Systems and, later, of the Distributed Control Systems Group. From 1996 to 2001, he acted as a Spanish Representative at the Council of the European Union Control Association. He is currently with the Department of Automatic Control, UPC. His research interests are in the areas of distributed, networked, and real-time control systems and applications. Since 1989, he has been coordinating projects at the national and international levels related to the aforementioned areas of expertise.

Dr. Fuertes is a member of the Administrative Committee of the IEEE Industrial Electronics Society, where he is the Chair of the Technical Committee on Networked-Based Control Systems and Applications. He has collaborated as the Organizer, the Chairman, the Session Organizer, and a member of program committees for several international conferences.

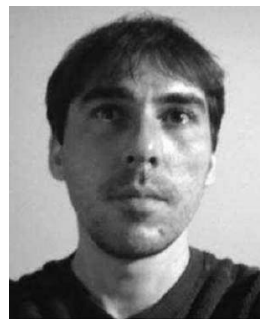

Antonio Camacho received the B.S. degree in chemical engineering and the M.S. degree in automation and industrial electronics from the Technical University of Catalonia, Barcelona, Spain, in 2000 and 2009, respectively, where he is currently working toward the Ph.D. degree in electronic engineering.

His research interests include networked and embedded control systems, industrial informatics, and power electronics.

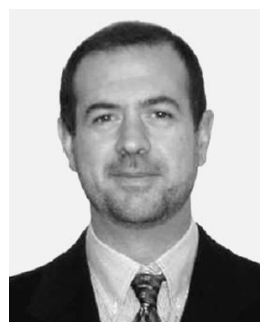

Giorgio Buttazzo (SM'05) received the B.S. degree in electronic engineering from the University of Pisa, Pisa, Italy, in 1985, the M.S. degree in computer science from the University of Pennsylvania, Philadelphia, in 1987, and the Ph.D. degree in computer engineering from Scuola Superiore Sant'Anna, Pisa, in 1991.

From 1987 to 1988 , he was with the General Robotics, Automation, Sensing and Perception Laboratory, University of Pennsylvania, where he worked on active perception and real-time control. $\mathrm{He}$ is currently a Full Professor of computer engineering with Scuola Superiore Sant'Anna. He has authored six books on real-time systems and over 200 papers in the field of real-time systems, robotics, and neural networks. His main research interests include real-time operating systems, dynamic scheduling algorithms, quality-of-service control, multimedia systems, advanced robotics applications, and neural networks. 